\title{
CrystEngComm
}

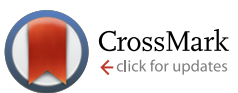

Cite this: CrystEngComm, 2015, 17, 824

Received 14th October 2014

Accepted 17th November 2014

DOI: 10.1039/c4ce02066a

www.rsc.org/crystengcomm

\section{Synthesis, structure determination, and formation of a theobromine : oxalic acid 2:1 cocrystal $\dagger$}

\author{
Franziska Fischer, ${ }^{\text {ab }}$ Gudrun Scholz, ${ }^{b}$ Lisa Batzdorf, ${ }^{\text {ab }}$ Manuel Wilke ${ }^{\text {ab }}$ \\ and Franziska Emmerling*a
}

\begin{abstract}
The structure and the formation pathway of a new theobromine: oxalic acid $(2: 1)$ cocrystal are presented. The cocrystal was synthesised mechanochemically and its structure was solved based on the powder X-ray data. The mechanochemical synthesis of this model compound was studied in situ using synchrotron XRD. Based on the XRD data details of the formation mechanism were obtained. The formation can be described as a self-accelerated ('liquid like') process from a highly activated species.
\end{abstract}

\section{Introduction}

The properties of active pharmaceutical ingredients (APIs) are typically not optimised for their planned applications. ${ }^{1-3}$ This is one of the crucial issues in the development of new pharmaceuticals. Many APIs show insufficient bioavailability, which is closely related to their low water solubility. In addition, the polymorphism of drugs has to be considered. Different modification of a given compound can be formed due to similar enthalpies of formation. The crystal structure of a material has a determining influence on its physicochemical properties including melting point, stability against physical and chemical stress, dissolution behaviour, solubility, and bioavailability. ${ }^{4}$

Polymorphs of an API can show changes in the properties or even in the therapeutic effects. ${ }^{5-7}$ Therefore, it remains a key challenge to improve the physicochemical properties of a drug. Crystal engineering considerations provide a possibility to overcome this issue. ${ }^{8}$ The formation of salts or solvates of APIs is a typical approach to circumvent the problem of low solubility. ${ }^{9-11}$ In this context, cocrystals of a given API have gained considerable interest in recent years. Cocrystals are crystalline phases with two or more components consisting of uncharged organic compounds, which interact via intermolecular forces. ${ }^{2,12-16}$ As a result of cocrystallisation, new crystal structures with new physicochemical properties compared to those of the API emerge. ${ }^{17-21}$

\footnotetext{
${ }^{a}$ BAM Federal Institute for Materials Research and Testing, Richard-WillstaetterStr. 11, 12489 Berlin, Germany. E-mail: franziska.emmerling@bam.de

${ }^{b}$ Department of Chemistry, Humboldt-Universität zu Berlin, Brook-Taylor-Str. 2, 12489 Berlin, Germany

$\dagger$ Electronic supplementary information (ESI) available: DTA-TGA measurements of the reactants and mass spectrometry coupled with DTA-TGA measurements of the cocrystal. CCDC 1028891. For ESI and crystallographic data in CIF or other electronic format see DOI: 10.1039/c4ce02066a
}

Cocrystals can be synthesised by different methods; typically solution based techniques are used. However, these methods present some disadvantages. For example, the solution based cocrystal formation requires a comparable solubility of the educts for successful synthesis. Due to the poor solubility of the APIs large amounts of solvent are needed. Additionally, solvent molecules could be incorporated in the crystalline structure of the cocrystal, which complicates the control of the product. ${ }^{3,22}$

Mechanochemistry is an elegant way to circumvent these problems. Typically no or only small amounts of solvent are needed for the milling reactions. Furthermore, the reactions are very fast, nearly quantitative, and proceed without the formation of by-products. Several cocrystals which are not accessible via conventional methods could only be synthesised mechanochemically. ${ }^{23-26}$ Consequently, mechanochemical syntheses have been used increasingly in the past years. A detailed understanding of the underlying mechanism of the mechanochemical syntheses is still scarce. ${ }^{27,28}$ Recently, Friščić et al. introduced real-time and in situ monitoring of milling reactions using a mixer mill. These experiments provided the first direct insight into the formation pathways. ${ }^{29-31}$ Here, we present the first in situ XRD study of milling synthesis using a ball mill setup.

As a model system, a new 2:1 cocrystal of the API theobromine (tb) and the coformer oxalic acid (ox) was used in the milling experiments. The in situ investigation of the reaction was conducted in a Perspex grinding jar using synchrotron XRD. Based on the obtained data, a diffusion mechanism was postulated.

\section{Results and discussion}

The tb:ox cocrystal in a molar ratio of $2: 1$ was synthesised by neat grinding. In contrast to the structurally similar APIs theophylline and caffeine only a few theobromine cocrystals 
are known..$^{3,32,33}$ The theobromine:oxalic acid (tb:ox) cocrystal represents an interesting model compound for further investigations of the formation pathway during the mechanochemical syntheses. The powder X-ray diffraction (PXRD) pattern of the new compound is depicted in Fig. 1a in comparison to the PXRD patterns of the reactants tb and ox dihydrate. The powder pattern of the cocrystal shows no contributions from the reflections of the reagents, indicating a complete reaction.

Based on the powder pattern determination of the cocrystal structure followed by Rietveld refinement was possible. The resulting structure is presented in Fig. 1b. The corresponding Rietveld refinement is shown in Fig. 2 indicating good agreement between the simulated and measured

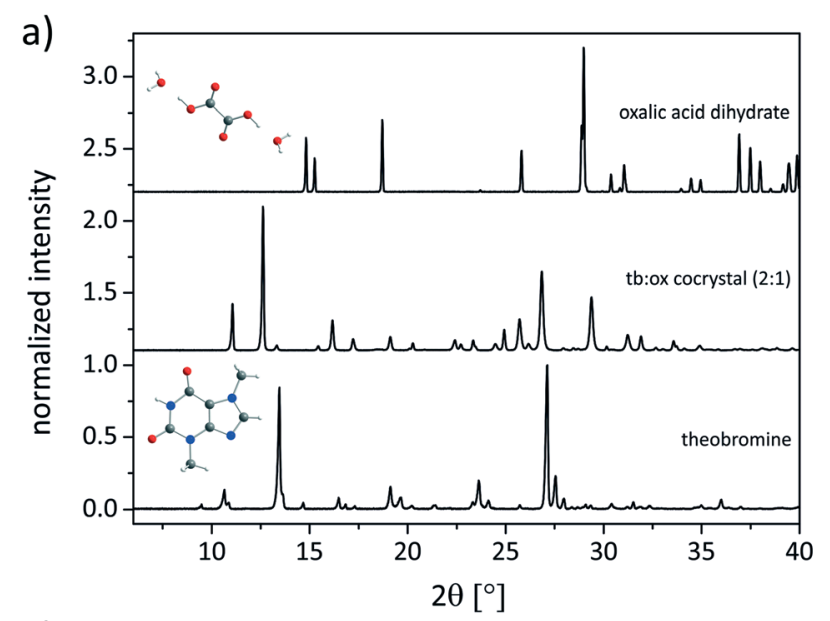

b)

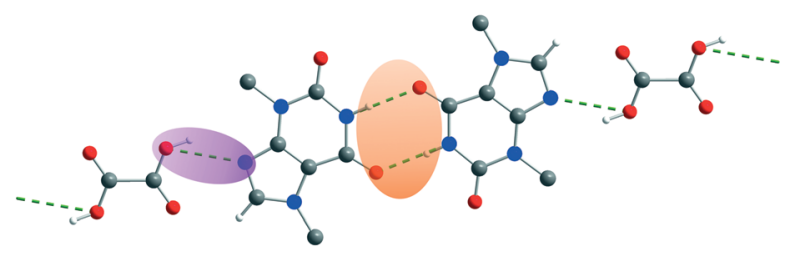

c)

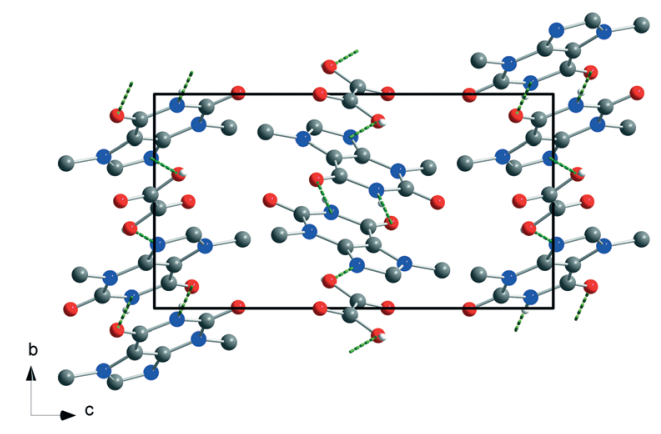

Fig. 1 a) Powder XRD patterns of the tb: ox cocrystal (center) and the reactants theobromine (bottom) and oxalic acid dihydrate (top). The background contributions of the sample holders were corrected. b) Bonding arrangement and c) structure of the 2:1 cocrystal tb:ox seen along the a-axis. The hydrogen atoms not involved in the hydrogen bonding were omitted for clarity. Green dashed lines indicate hydrogen bonds. b) The structure of the $2: 1$ cocrystal tb: ox seen along the a-axis. The hydrogen atoms not involved in the hydrogen bonding were omitted for clarity. Green dashed lines indicate hydrogen bonds.

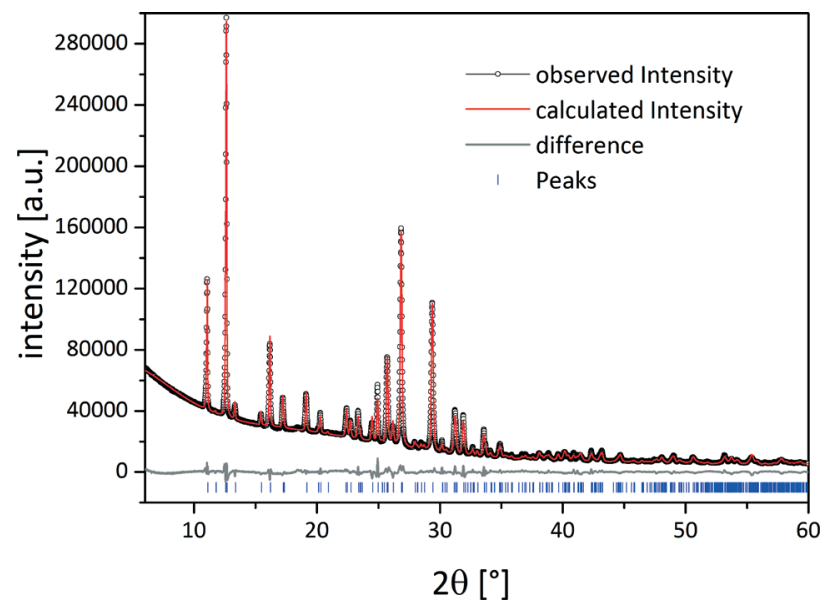

Fig. 2 Rietveld refinement of the crystal structure of the tb:ox cocrystal. Scattered X-ray intensity for the tb:ox 2:1 cocrystal under ambient conditions as a function of the diffraction angle $2 \theta$. The observed pattern (black circles), the best Rietveld fit profile (red line), the reflection positions (blue tick marks), and the difference curve (grey line) between the observed and calculated profiles are shown. The wavelength was $\lambda=1.54056 \AA\left(\mathrm{Cu} \mathrm{K} \alpha_{1}\right)$. The $R$-values are $R_{\mathrm{p}}=$ $3.2 \%$ and $R_{\mathrm{wp}}=4.9 \% ; R_{\mathrm{p}}$, and $R_{\mathrm{wp}}$ refer to the Rietveld criteria of fit for profile and weighted profile defined by Langford and Louer. ${ }^{34}$

powder patterns. The tb:ox cocrystal crystallises in the monoclinic space group $P 2_{1} / c(a=8.89209(45) \AA, \quad b=$ $7.50930(28) \AA, c=15.60777(84) \AA, \beta=116.5691(38)^{\circ}, V=$ 932.124(83) $\left.\AA^{3}\right)$. Each tb molecule is connected to a tb molecule and an ox molecule via hydrogen bonds. One hydrogen bond is formed between the nitrogen atom of the secondary amine of a tb molecule and the oxygen atom from a carbonyl group of another tb molecule $\left(\mathrm{N}-\mathrm{H} \cdots \mathrm{O}, d_{\mathrm{H} \cdots \mathrm{A}}=2.803 \AA\right.$, $\left.d_{\mathrm{D} \cdots \mathrm{A}}=1.92 \AA, \angle \mathrm{D}-\mathrm{H} \cdots \mathrm{A}=170^{\circ}\right)$, resulting in an $\mathrm{R}_{2}^{2}(8)$ dimer (orange). An additional hydrogen bond (violet) is formed between the tertiary amine of a tb molecule and the hydroxyl group of an ox carboxyl group $\left(\mathrm{O}-\mathrm{H} \cdots \mathrm{N}, d_{\mathrm{H} \cdots \mathrm{A}}=2.843 \AA\right.$, $d_{\mathrm{D} \cdots \mathrm{A}}=2.12 \AA, \angle{ }_{\mathrm{D}-\mathrm{H} \cdots \mathrm{A}}=136^{\circ}$ ), leading to a twisted chain motif running along the $b$-axis.

The absence of water in the crystal structure is evident from the DTA-TGA measurements (Fig. S3 $\dagger$ ). The first DTA signal of the cocrystal arises at a temperature of $252{ }^{\circ} \mathrm{C}$. At this temperature the ox molecules decompose. Since the decomposition temperature of ox is $50 \mathrm{~K}$ higher than that of the pure sample, it can be concluded that the ox molecules are stabilised in the cocrystal.

Based on PXRD data, the position of the hydrogen atoms cannot be determined unambiguously. In order to exclude salt formation the cocrystal was investigated by Raman (Fig. S1 $\dagger$ ) and solid-state NMR (ssNMR) spectroscopy (Fig. S2†). In the Raman spectra only the band attributed to the carboxylate deformation vibration of ox dihydrate at $478 \mathrm{~cm}^{-1}$ shows a pronounced shift. ${ }^{35}$ Therefore protonation of the tb molecules in the cocrystal can be excluded. The strong shift of the carboxylate band indicates that the ox molecules interact more strongly with water in ox dihydrate than with the tb molecule in the cocrystal. This assumption is supported by 
the ssNMR measurements. The only ssNMR signal, which shifts considerably in the cocrystal, is due to the protons of the ox molecules at $\mathbf{1 7 . 0} \mathrm{ppm}$. The shift to $14.2 \mathrm{ppm}$ suggests that the protons of ox are not as strongly bridged in the cocrystal as in pure ox dihydrate. Consequently, it can be assumed that the ox molecules are uncharged in the cocrystal. The water signal at $5.5 \mathrm{ppm}$ disappears in the cocrystal, which reveals that no water molecules are incorporated in the cocrystal. Moreover, the observed line broadening in the ${ }^{1} \mathrm{H}$ MAS NMR spectrum of the cocrystal (Fig. S2, $\dagger$ middle) supports the assumption of the formation of a network of additional hydrogen bonds.

The formation of the cocrystal was observed with in situ synchrotron XRD. The in situ experiments were conducted at the microfocus beamline $\mu$ Spot (BESSY II, Helmholtz Centre Berlin for Materials and Energy, Germany) in transmission geometry using a wavelength of 1.0000 Å. A Perspex grinding jar (Fig. 3) was used as the reaction vessel. Powder diffraction patterns of the reaction mixture can be measured directly without opening the grinding jar. ${ }^{36}$ XRD patterns were acquired every $30 \mathrm{~s}$ during the milling process.

An investigation of the mechanochemical cocrystal formation pathway was possible since the powder patterns of the cocrystal and the reactants revealed highly distinguishable, characteristic reflections. Fig. 4 shows the time resolved powder patterns obtained during the neat grinding of tb and ox dihydrate over a time span of 20 minutes. The milling reaction can be divided into three phases. In the first step only the reflections of the reactants $t b$ and ox dihydrate are observed in the XRD patterns (phase 1). In the first $12 \mathrm{~min}$ the continuous, slow decomposition of the crystal structure of the reactants is traceable on the basis of the decreasing intensity of the tb reflection at $13.5^{\circ}$ (Fig. S8 $\dagger$ ). Afterwards the fast formation of the cocrystal proceeds within $60 \mathrm{~s}$ (phase 2). In this second phase the reflections of the reactants are still detectable and decrease quickly with prolonged milling times. Neither the formation of a transient intermediate species nor prolonged amorphisation of the reaction mixture could be observed during this phase. The last phase begins at a milling time of $13.5 \mathrm{~min}$. At this point there are no crystalline educts detectable in the reaction mixture (phase 3).

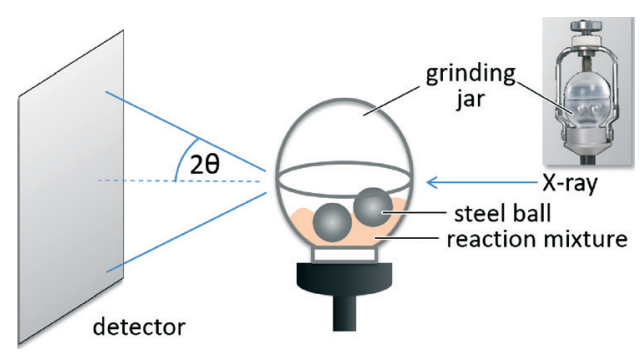

Fig. 3 Experimental setup for the collection of the powder patterns during the neat grinding synthesis of the tb: ox cocrystal. The sketch shows a Perspex grinding jar filled with two $10 \mathrm{~mm}$ stainless steel grinding balls.

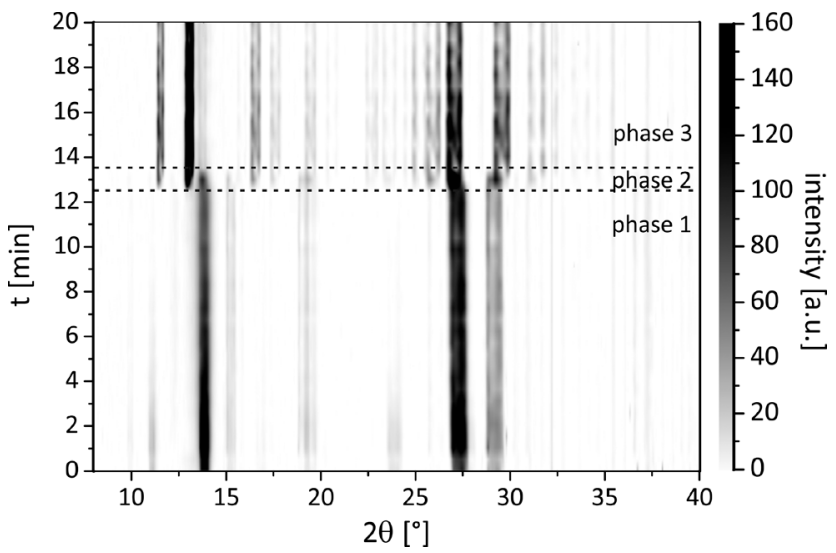

Fig. 4 Time resolved pathway of the powder patterns during the neat grinding synthesis of the tb: ox cocrystal.

Different explanations for the mechanochemical reactions are discussed in the literature and three theories have to be considered: i) the hot spot theory, ii) the magma-plasma model, and iii) reaction via diffusion. The hot spot theory is based on the assumption that the attrition between the surfaces causes local temperatures to rise above $1000{ }^{\circ} \mathrm{C}$ for short periods $\left(10^{-3}\right.$ to $\left.10^{-4} \mathrm{~s}\right)$ on a molecular dimension. ${ }^{37-39}$ The magma-plasma model involves local temperatures about $10^{4}{ }^{\circ} \mathrm{C}$ leading to transient plasma and the ejection of energy. ${ }^{37,40}$ The third approach emphasises the importance of short diffusion pathways driven by excellent mixing of the reactants and accelerated reactions. ${ }^{41}$ No clear indication of a mechanism based on one of the three models could be found for the investigated synthesis. Keeping in mind that the diffusion coefficient in the solid state $\left(D \approx 10^{-16} \mathrm{~m}^{2} \mathrm{~s}^{-1}\right)$ is significantly lower than the diffusion coefficient in fluid phases $\left(D \approx 10^{-9} \mathrm{~m}^{2} \mathrm{~s}^{-1}\right.$ ) a comparison of the conditions during milling with a liquid-like situation appears obvious.

The fast transformation can be explained by a selfaccelerated process from a highly activated species that is completed in $60 \mathrm{~s}$. This process leads to the direct conversion of the solid reactants to the product. There is no driving force based on salt formation or protonation since the cocrystal consists only of neutral molecules. The derived formation pathway is illustrated in Fig. 5.

\section{Experimental}

\section{Materials}

Theobromine, $\mathrm{C}_{7} \mathrm{H}_{8} \mathrm{~N}_{4} \mathrm{O}_{2}$, (99\%, Acros Organics, Belgium) and oxalic acid dihydrate, $\mathrm{C}_{2} \mathrm{H}_{2} \mathrm{O}_{4} \cdot 2 \mathrm{H}_{2} \mathrm{O},(\geq 99+\%$, Acros Organics, Belgium) were purchased commercially and were used without further purification.

\section{Milling synthesis}

The synthesis of the title compound was conducted by neat grinding in a ball mill (MM400, Retsch, Germany) at $30 \mathrm{~Hz}$ for $25 \mathrm{~min}$ in a molar ratio of 1.9:1 theobromine : oxalic acid 


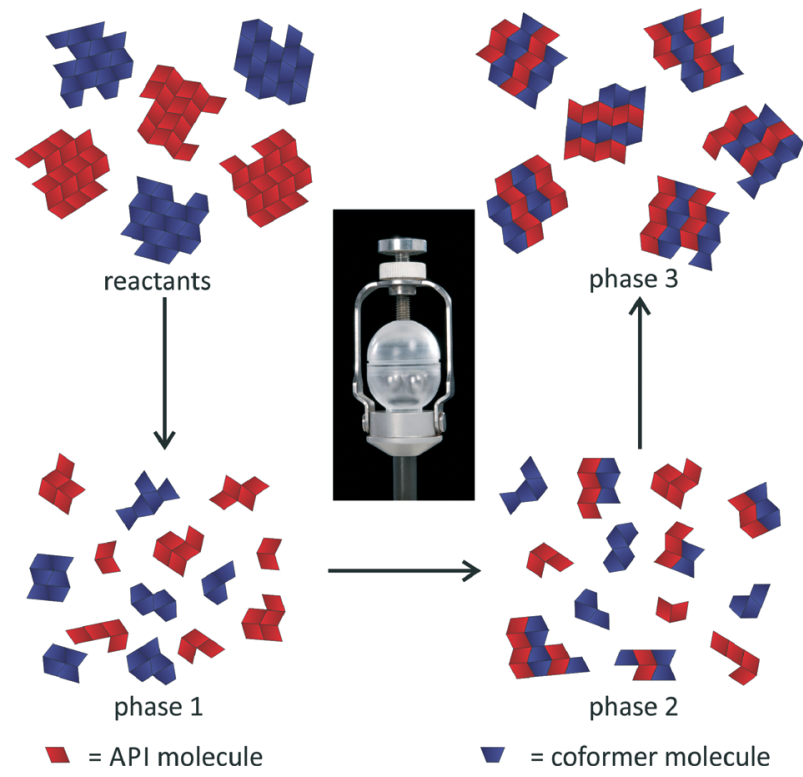

Fig. 5 Representation of the mechanism during the milling synthesis of the $2: 1$ cocrystal of tb and ox.

dihydrate. A $10 \mathrm{~mL}$ steel vessel with two steel balls $(10 \mathrm{~mm})$ was used for a total load of $1 \mathrm{~g}$.

\section{XRD measurements}

The obtained product was investigated by PXRD. The obtained powder pattern did not show any residues of the reagents. All X-ray diffraction experiments were carried out using a D8 diffractometer (Bruker AXS, Karlsruhe, Germany) in transmission geometry $\left(\mathrm{Cu}-\mathrm{K}_{1}\right.$ radiation, $\lambda=1.54056 \AA$ ). The structure was solved based on the PXRD pattern using the open source program FOX for indexing and calculating the structure. ${ }^{42}$ The program CHEKCELL was used to confirm the unit cell and the space group. ${ }^{43}$ FOX uses global optimisation algorithms to solve the structure by performing trials in direct space. This search algorithm uses random sampling coupled with simulated temperature annealing to locate the global minimum of the figure-of-merit factor. To reduce the total number of degrees of freedom, the theobromine molecule was treated as rigid. The crystal structure of the cocrystal was solved by the simulated annealing procedure on a standard personal computer within $12 \mathrm{~h}$ finding the deepest minimum of the cost function several times during the procedure. To complete the structure determination, the structural solution obtained from Monte Carlo/simulated annealing was subsequently subjected to Rietveld refinement employing the TOPAS software. ${ }^{44}$ CCDC 1028891 contains the supplementary crystallographic data for the tb: ox $2: 1$ cocrystal.

\section{Synchrotron measurements}

In situ measurements were performed at the microfocus beamline $\mu$ Spot (BESSY II, Helmholtz Centre Berlin for Materials and Energy, Germany) in transmission geometry. The powder patterns were collected at a wavelength of $1.0000 \AA$ using a Si (111) double-crystal monochromator. A twodimensional MarMosaic CCD X-ray detector with $3072 \times 3072$ pixels was used to record the scattering intensity. In a typical experiment, the XRD patterns were collected every $10 \mathrm{~s}$. The obtained scattering images were processed and converted into diagrams of scattered intensities versus the scattering vector $q(q=4 \pi / \lambda \sin \theta)$ employing an algorithm from the FIT2D software. ${ }^{45}$ For the graphical representations, the $q$ values were transformed into the diffraction angle $2 \theta(\mathrm{Cu})$ to provide a direct comparison to the results obtained by XRD experiments performed with $\mathrm{Cu}$ radiation. The in situ monitoring of the synthesis of the title compound was conducted by neat grinding in a Mini-Mill PULVERISETTE 23 (Fritsch, Germany) at $30 \mathrm{~Hz}$ for $20 \mathrm{~min}$ in a molar ratio of $1.9: 1$ theobromine: oxalic acid dihydrate. A $10 \mathrm{~mL}$ self-constructed Perspex vessel with two steel balls $(10 \mathrm{~mm})$ was used for a total load of $1 \mathrm{~g}$. Every $30 \mathrm{~s}$ of milling a powder pattern of the sample was taken.

\section{Raman spectroscopy}

Raman measurements were performed on a Raman RXN1 $1^{\mathrm{TM}}$ analyser (Kaiser Optical Systems, France). The spectra were collected using a laser with a wavelength of $\lambda=785 \mathrm{~nm}$ and a contactless probe head (working distance: $1.5 \mathrm{~cm}$, spot size: $1.0 \mathrm{~mm}$ ). The Raman spectra were recorded with an acquisition time of $5 \mathrm{~s}$ and 5 accumulations. NIR excitation radiation at $\lambda=785 \mathrm{~nm}$ and irradiation of $6.6 \mathrm{~W} \mathrm{~cm} \mathrm{~cm}^{-2}$ were performed.

\section{SSNMR spectroscopy}

${ }^{1} \mathrm{H}$ magic angle spinning (MAS) NMR spectra were recorded on a Bruker AVANCE 400 spectrometer using a $2.5 \mathrm{~mm}$ double-bearing MAS probe (Bruker BioSpin), applying a spinning speed of $20 \mathrm{kHz}$. The ${ }^{1} \mathrm{H}$ MAS NMR spectra were recorded with a $\pi / 2$ pulse length of $3.6 \mu \mathrm{s}$, a recycle delay of $5 \mathrm{~s}$ and an accumulation number of 256. Existent background signals were suppressed with a phase-cycled depth pulse sequence according to Cory and Ritchey. ${ }^{46}$

\section{DTA and TGA measurements}

DTA and TGA measurements were conducted using a thermobalance SETARAM TAG24 in $1600{ }^{\circ} \mathrm{C}$ equipment. The measurements were performed in an open $\mathrm{Pt}$ jar under $\mathrm{N}_{2}$ /synthetic air flow with a heating rate of $10 \mathrm{~K} \mathrm{~min}^{-1}$. No cycle measurements were taken.

\section{Conclusions}

The crystal structure of a tb cocrystal with ox in a $2: 1$ ratio was solved from powder diffraction data. The cocrystal was synthesised mechanochemically. Due to the extremely poor solubility of tb this cocrystal could not be obtained from solution. Based on the Raman spectroscopy and SSNMR data the formation of a salt could be excluded. The synthesis 
pathway was investigated using in situ XRD and a three step mechanism was derived. The experiment proved that this approach is feasible for the characterisation of mechanochemical reactions.

\section{Acknowledgements}

We are grateful to Dr. S. Reinsch (BAM) for DTA-TGA measurements.

\section{Notes and references}

1 O. Almarsson and M. J. Zaworotko, Chem. Commun., 2004, 1889-1896.

2 S. Aitipamula, R. Banerjee, A. K. Bansal, K. Biradha, M. L. Cheney, A. R. Choudhury, G. R. Desiraju, A. G. Dikundwar, R. Dubey, N. Duggirala, P. P. Ghogale, S. Ghosh, P. K. Goswami, N. R. Goud, R. R. K. R. Jetti, P. Karpinski, P. Kaushik, D. Kumar, V. Kumar, B. Moulton, A. Mukherjee, G. Mukherjee, A. S. Myerson, V. Puri, A. Ramanan, T. Rajamannar, C. M. Reddy, N. Rodríguez-Hornedo, R. D. Rogers, T. N. G. Row, P. Sanphui, N. Shan, G. Shete, A. Singh, C. C. Sun, J. A. Swift, R. Thaimattam, T. S. Thakur, R. Kumar Thaper, S. P. Thomas, S. Tothadi, V. R. Vangala, N. Variankaval, P. Vishweshwar, D. R. Weyna and M. J. Zaworotko, Cryst. Growth Des., 2012, 12, 2147-2152.

3 H. D. Clarke, K. K. Arora, H. Bass, P. Kavuru, T. T. Ong, T. Pujari, L. Wojtas and M. J. Zaworotko, Cryst. Growth Des., 2010, 10, 2152-2167.

4 N. Schultheiss and A. Newman, Cryst. Growth Des., 2009, 9, 2950-2967.

5 B. Sarma, J. Chen, H. Y. Hsi and A. S. Myerson, Korean J. Chem. Eng., 2011, 28, 315-322.

6 J. Bauer, S. Spanton, R. Henry, J. Quick, W. Dziki, W. Porter and J. Morris, Pharm. Res., 2001, 18, 859-866.

7 J.-P. Brog, C.-L. Chanez, A. Crochet and K. M. Fromm, RSC Adv., 2013, 3, 16905-16931.

8 G. R. Desiraju, Angew. Chem., Int. Ed., 2007, 46, 8342-8356.

9 Y. Umeda, T. Fukami, T. Furuishi, T. Suzuki, K. Tanjoh and K. Tomono, Drug Dev. Ind. Pharm., 2009, 35, 843-851.

10 A. T. M. Serajuddin, Adv. Drug Delivery Rev., 2007, 59, 603-616.

11 R. Chadha, A. Saini, P. Arora and S. Bhandari, Crit. Rev. Ther. Drug Carrier Syst., 2012, 29, 183-218.

12 C. B. Aakeroy, M. E. Fasulo and J. Desper, Mol. Pharmaceutics, 2007, 4, 317-322.

13 M. C. Etter and G. M. Frankenbach, Chem. Mater., 1989, 1, 10-12.

14 T. Friščić and W. Jones, J. Pharm. Pharmacol., 2010, 62, 1547-1559.

15 C. B. Aakeröy, M. E. Fasulo and J. Desper, Mol. Pharmaceutics, 2007, 4, 317-322.

16 M. J. Zaworotko, Cryst. Growth Des., 2006, 7, 4-9.
17 D. P. McNamara, S. L. Childs, J. Giordano, A. Iarriccio, J. Cassidy, M. S. Shet, R. Mannion, E. O'Donnell and A. Park, Pharm. Res., 2006, 23, 1888-1897.

18 D. J. Good and N. R. Rodríguez-Hornedo, Cryst. Growth Des., 2009, 9, 2252-2264.

19 A. V. Trask, W. D. S. Motherwell and W. Jones, Int. J. Pharm., 2006, 320, 114-123.

20 A. V. Trask, W. D. S. Motherwell and W. Jones, Cryst. Growth Des., 2005, 5, 1013-1021.

21 N. Chieng, M. Hubert, D. Saville, T. Rades and J. Aaltonen, Cryst. Growth Des., 2009, 9, 2377-2386.

22 J. S. Stevens, S. J. Byard, C. A. Muryn and S. L. M. Schroeder, J. Phys. Chem. B, 2010, 114, 13961-13969.

23 S. L. James, C. J. Adams, C. Bolm, D. Braga, P. Collier, T. Friščić, F. Grepioni, K. D. Harris, G. Hyett and W. Jones, Chem. Soc. Rev., 2012, 41, 413-447.

24 D. Braga, L. Maini, M. Polito and F. Grepioni, Chem. Commun., 2002, 2302-2303.

25 S. Karki, T. Friscic and W. Jones, CrystEngComm, 2009, 11, 470-481.

26 V. R. Pedireddi, W. Jones, A. P. Chorlton and R. Docherty, Chem. Commun., 1996, 987-988.

27 M. R. Caira, L. R. Nassimbeni and A. F. Wildervanck, J. Chem. Soc., Perkin Trans. 2, 1995, 2213-2216.

28 M. C. Etter, S. M. Reutzel and C. G. Choo, J. Am. Chem. Soc., 1993, 115, 4411-4412.

29 T. Friščić, I. Halasz, P. J. Beldon, A. M. Belenguer, F. Adams, S. A. J. Kimber, V. Honkimäki and R. E. Dinnebier, Nat. Chem., 2013, 5, 66-73.

30 I. Halasz, S. A. J. Kimber, P. J. Beldon, A. M. Belenguer, F. Adams, V. Honkimäki, R. C. Nightingale, R. E. Dinnebier and T. Friščić, Nat. Protoc., 2013, 8, 1718-1729.

31 I. Halasz, A. Puškarić, S. A. J. Kimber, P. J. Beldon, A. M. Belenguer, F. Adams, V. Honkimäki, R. E. Dinnebier, B. Patel, W. Jones, V. Štrukil and T. Friščić, Angew. Chem., Int. Ed., 2013, 52, 11538-11541.

32 S. Karki, L. Fábián, T. Friščić and W. Jones, Org. Lett., 2007, 9, 3133-3136.

33 N. Madusanka, M. D. Eddleston, M. Arhangelskis and W. Jones, Acta Crystallogr., Sect. B: Struct. Sci., Cryst. Eng. Mater., 2014, 70, 72-80.

34 J. I. Langford and D. Louer, Rep. Prog. Phys., 1996, 59, 131-234.

35 Y. Ebisuzaki and S. M. Angel, J. Raman Spectrosc., 1981, 11, 306-311.

36 L. Batzdorf, F. Fischer, M. Wilke, K. J. Wenzel and F. Emmerling, Angew. Chem., Int. Ed., 2014, DOI: 10.1002/anie.201409834R1.

37 P. Baláž, in Mechanochemistry in Nanoscience and Minerals Engineering, Springer, 2008, pp. 1-102.

38 F. P. Bowden and D. Tabor, The friction and lubrication of solids, Clarendon Press, Oxford, 1958.

39 F. P. Bowden and A. D. Yoffe, The Initiation and Growth of Explosion in Liquids and Solids, CUP Archive, 1952.

40 P. Thiessen, Abh. Dtsch. Akad. Wiss. Berlin, Kl. Chem., Geol. Biol., 1966, 15.

41 X. Ma, W. Yuan, S. E. J. Bell and S. L. James, Chem. Commun., 2014, 50, 1585-1587. 
42 V. Favre-Nicolin and R. Cerny, J. Appl. Crystallogr., 2002, 35, 734-743.

43 J. Laugier and B. Bochu, Chekcell, 2001.

44 Topas Version 2.0, General Profile and Structure Analysis Software for Powder Diffraction Data (User Manual), Bruker AXS, Karlsruhe (Germany), 2000.
45 A. P. Hammersley, K. Brown, W. Burmeister, L. Claustre, A. Gonzalez, S. McSweeney, E. Mitchell, J.-P. Moy, S. O. Svensson and A. W. Thompson, J. Synchrotron Radiat., 1997, 4, 67-77.

46 D. G. Cory and W. M. Ritchey, J. Magn. Reson., 1988, 80, 128-132. 